\title{
Confiabilidad de la sectorización óptima de redes de distribución de agua
}

\section{Reliability of optimal partitioning of water distribution networks}

Presentación: 06-07/10/20

\section{Doctorando:}

\section{Melina Denardi}

Laboratorio de Métodos y Simulaciones Computacionales, Universidad Tecnológica Nacional, Facultad Regional Rafaela, Acuña 49, 2300 Rafaela, Argentina.

ingmdenardi@gmail.com

\section{Director/a:}

\section{Gabriel D. Puccini}

\section{Co-director/a:}

\section{Resumen}

La sectorización óptima de redes de agua potable es uno de los enfoques innovadores para el análisis y gestión de los sistemas hidráulicos que más se ha potenciado en los últimos años. Abarca aspectos técnicos como la planificación de sistemas de medición eficientes, la creación de zonas de control de presión, la instalación de instrumentos para el monitoreo y la detección temprana de contaminantes. En este trabajo se evalúa la confiabilidad de la sectorización óptima frente a un aumento de las demandas de un número determinado de consumidores. Se analiza la sectorización obtenida maximizando el índice de modularidad y se la contrapone con la red sin segmentar. Conjuntamente, se realiza una comparación con la estructura obtenida maximizando una función objetivo que combina la modularidad y la resiliencia de la red. El procedimiento se aplica a dos sistemas hidráulicos: una red académica de 25 nodos y una red real de la Provincia de Córdoba.

Palabras clave: Confiabilidad; Resiliencia; Modularidad; Segmentación

\begin{abstract}
Optimal partitioning of water distribution networks is a novel approach for the analysis and management of hydraulic systems. It covers technical aspects such as metering water consumption, control of pressure and leakages, location of isolation valves, and early contaminant detection. In this work, optimal partitioning reliability is evaluated by considering demand increments for a given number of consumers. First, the partitioning is obtained by maximizing the modularity index, and then, the resulting communities are analyzed and contrasted with the original unsegmented network. In addition, a comparison is performed with the structure obtained by maximizing an objective function which combines the modularity and resilience of the network. The procedure is applied to two hydraulic systems: an academic network of 25 nodes and the Córdoba Province network.
\end{abstract}

Keywords: Reliability; Resilience; Modularity; Segmentation 


\section{Introducción}

Durante el siglo XIX las redes de distribución de agua se diseñaban utilizando un enfoque que buscaba satisfacer las demandas de los consumidores manteniendo la presión nodal requerida. En la actualidad esas redes suelen experimentar fugas y fallas periódicas debido al incremento en la demanda de los consumidores y al deterioro de sus componentes. Así, surgen nuevos desafíos en el diseño de la expansión y la gestión de estos sistemas complejos. Por ejemplo, no existe un método que permita determinar la ubicación de las fugas que, en algunas redes muy antiguas, pueden representar hasta el 70\% del servicio de agua (Farley, 2001), o evitar la caída de las presiones nodales por debajo del nivel mínimo requerido. La gestión de los sistemas de distribución de agua es un proceso complejo debido a los diferentes aspectos que intervienen, como la conectividad de la red y la ubicación de las fuentes, válvulas o bombas; además, de la compleja topología de las redes que fueron diseñadas usando criterios tradicionales.

Uno de los enfoques innovadores para el análisis y la gestión de estos sistemas que más se ha potenciado en los últimos años, especialmente en los países anglosajones y en Italia, es la división de la red de distribución en distritos más pequeños mediante la instalación de válvulas de aislación y caudalímetros en tubos óptimamente seleccionados (Castro-Gama et al., 2016; Liu et al., 2018). La posibilidad de sectorizar una red de manera óptima abarca aspectos técnicos fundamentales como la planificación de sistemas de medición eficientes, la creación de zonas de control de presión, la colocación de válvulas y la instalación de sistemas de monitoreo para la detección temprana de contaminantes (Laucelli et al., 2017).

Se han propuesto distintas técnicas para la sectorización óptima de las redes de distribución de agua aunque todavía no existe un acuerdo universal. Desde la teoría de redes complejas, Newman y Girvan (2004) introdujeron el concepto de modularidad como una medida de la estructura en comunidades o módulos que posee una red. Así, las redes con alta modularidad poseen una densa conectividad entre los nodos dentro de cada módulo, pero escasas conexiones entre nodos de diferentes módulos. Sin embargo, el uso directo de este índice que ha sido definido sobre la base de consideraciones netamente topológicas, no está totalmente aceptado en los sistemas hidráulicos debido a que las redes de agua son fuertemente dependientes de parámetros físicos como las demandas, los diámetros de las tuberías o las presiones nodales. Además, se desconoce cómo se ve afectada la modularidad al incluir en la optimización el concepto de resiliencia, el cual se define como capacidad que tiene el sistema de distribución de agua para sobrellevar una falla o incrementos en las demandas como, por ejemplo, consecuencia del crecimiento de la población.

En este trabajo se evalúa la confiabilidad de la sectorización midiendo el impacto que ocasiona un aumento de las demandas sobre diferentes estructuras de comunidades. Se estudia si el diseño de la sectorización es capaz de generar soluciones estables frente a diferentes patrones de incrementos de las demandas de los consumidores. Este procedimiento se aplicó a una red académica compuesta por 25 nodos, y luego a una red real de una localidad de la provincia de Córdoba, con 431 nodos.

\section{Desarrollo}

\section{Índice de modularidad}

La teoría de redes complejas provee un conjunto de herramientas potentes para caracterizar la estructura en comunidades de los sistemas de distribución de agua. El índice de modularidad es la métrica más ampliamente utilizada (Newman y Girvan, 2004) y está dado por:

$$
\mathrm{Q}=\frac{1}{2 \mathrm{n}_{\mathrm{p}}} \sum_{\mathrm{ij}}\left(\mathrm{A}_{\mathrm{ij}}-\frac{\mathrm{k}_{\mathrm{i}} \mathrm{k}_{\mathrm{j}}}{2 \mathrm{n}_{\mathrm{p}}}\right) \delta\left(\mathrm{M}_{\mathrm{i}}, \mathrm{M}_{\mathrm{j}}\right)
$$

donde $\mathrm{A}_{\mathrm{ij}}$ son los elementos de la matriz de adyacencia $A$, y $k_{i}$ es el grado del nodo $i$, es decir, el número de tubos conectados al nodo $i, M_{i}$ identifica el módulo $i$ de la red, $\delta$ es la función delta de Kronecker $\left(\delta=1\right.$ sólo si $M_{i}=M_{j}$ ) y la suma corre sobre 
todos los posibles pares de nodos $(i, j)$, con $i \neq j$. Básicamente, este índice identifica los grupos de nodos que tienen alta densidad de conexión entre ellos, y con menor densidad de conexión entre grupos. Así, el índice de modularidad $Q$ es una medida de la capacidad que tiene la red a la división en módulos o comunidades basado solamente en propiedades de conectividad.

\section{Índice de resiliencia}

El concepto de resiliencia fue introducido por Todini (2000) como una métrica que permite cuantificar el rendimiento del sistema hidráulico, y se usa habitualmente como una medida de la confiabilidad del sistema. Además, cuando es usado en procesos de optimización, permite mejorar el rendimiento del sistema bajo condiciones de falla proporcionando mayor potencia que la mínima requerida en cada nodo. El índice de resiliencia se define como:

$$
\mathrm{I}_{\mathrm{R}}=\frac{\sum_{\mathrm{i}=1}^{\mathrm{N}_{\mathrm{n}}} \mathrm{q}_{\mathrm{i}}^{*}\left(\mathrm{~h}_{\mathrm{i}}-\mathrm{h}_{\mathrm{i}}^{*}\right)}{\sum_{\mathrm{k}=1}^{\mathrm{N}_{\mathrm{r}}} \mathrm{Q}_{\mathrm{k}} \mathrm{H}_{\mathrm{k}}-\sum_{\mathrm{i}=1}^{\mathrm{N}_{\mathrm{n}}} \mathrm{q}_{\mathrm{i}}^{*} \mathrm{~h}_{\mathrm{i}}^{*}}
$$

donde $q_{i}^{*}$ y $h_{i}^{*}$ denotan la demanda y presión requerida en el nodo $i$. Asimismo, $q_{i}$ y $h_{i}$ son, respectivamente, la demanda y la presión actual de cada nodo $i, N_{n}$ es el número de nodos, $Q_{k}$ y $H_{k}$ son la descarga y la presión, respectivamente, de cada reservorio $k$, siendo $N_{r}$ el número de reservorios, y $\gamma$ el peso específico del agua. Considerando que el índice propuesto está basado en un análisis del flujo de la red en estado estacionario y en la energía disipada en las tuberías, se desprende que el índice de resiliencia de una red es una medida del excedente de energía disponible para el abastecimiento (Herrera et al., 2015). De esta manera, un alto índice de resiliencia permite tener una red con mayor capacidad para sobrellevar fallas hidráulicas.

\section{Formulación del problema de optimización}

El problema de la sectorización óptima de una red de distribución de agua consiste en la división de la red original en redes más pequeñas, llamadas comunidades, cada una de las cuales debe poseer la capacidad de funcionar de manera autónoma. La función objetivo para la estrategia de sectorización propuesta está dada por:

$$
C(x)=\lambda Q(x)+(1-\lambda) I_{R}(x)
$$

donde $\mathrm{x}=\left[\mathrm{x}_{1}, \ldots, \mathrm{x}_{\mathrm{N}_{\mathrm{p}}}\right]$ es un vector binario, con la dimensión $\mathrm{N}_{\mathrm{p}}$ del número de tubos, que codifica la conectividad entre nodos, y $\lambda$ es el parámetro de sintonía. De modo que el problema de optimización se escribe:

$$
\begin{aligned}
& \max \mathrm{C}(\mathrm{x}) \\
& \text { s.a.: } h_{i} \geq h_{i}^{*} \quad i=1, \ldots, N_{n}
\end{aligned}
$$

Con el objeto de obtener segmentaciones que representen soluciones factibles, el algoritmo propuesto resuelve el sistema hidráulico para cada posible solución x. Si alguna de estas soluciones posee nodos cuyas presiones son menores que la presión mínima, o presiones negativas, a la función de costo se le aplica una penalización $\mathrm{P}=\sum_{\mathrm{i}=1}^{\mathrm{N}_{\mathrm{n}}} \min \left(0, \mathrm{~h}_{\mathrm{i}}-\mathrm{h}_{\mathrm{i}}^{*}\right)$. Esta estrategia permite disminuir de forma desproporcionada el costo de soluciones no factibles, alejándolas del espacio de búsqueda del algoritmo. Nótese que si la restricción de las presiones mínimas es satisfecha, la función de costo C toma valores en el intervalo (0.1).

El problema de la sectorización óptima de las redes de agua es un problema combinatorio del tipo NP-completo. En el presente trabajo se implementa un algoritmo de recocido simulado (Kirkpatrick et al., 1983), aunque los resultados obtenidos son independientes del algoritmo utilizado. 
En este trabajo se analizan los resultados de la sectorización obtenidos a partir de la maximización de C para dos escenarios distintos. El primer escenario se establece para $\lambda=1.0$. Así, la sectorización consiste en la maximización del índice de modularidad Q, lo que implica obtener el mayor número de módulos que sean similares respecto a su conectividad. El segundo escenario se obtiene para $\lambda=0.5$; de modo que se busca sectorizar la red en comunidades con máxima modularidad pero compatible con la máxima resiliencia del sistema. El análisis de confiabilidad del sistema bajo incrementos en las demandas se realiza luego del proceso de sectorización. En la Figura 2 (primer caso de estudio) se perturban las demandas en un número fijo de $\operatorname{nodos}(\mathrm{N}=1$ y N=10) multiplicándolas por un factor $\alpha$ que varía entre 1 y 2, con incrementos de 0.1. En la Figura 4 (segundo caso de estudio), se perturban las demandas de $\mathrm{N}=20$ y N=50 nodos y el factor $\alpha$ varía entre 1 y 4 , con incrementos de 0.25 . Para cada incremento se realizan 300 iteraciones escogiendo aleatoriamente los nodos cuyas demandas se van a modificar. El valor de resiliencia de cada perturbación se promedia para las 300 iteraciones.

\section{Casos de estudio}

Para evaluar la confiablidad de la sectorización se analizan dos sistemas diferentes. El primero de ellos, consiste de una red de estudio académico compuesta por 25 nodos, 40 tubos y 4 tanques; no posee diferencia de cota y su estructura se puede observar en la Figura 1. En la Figura 1-izquierda, los nodos con demandas iguales se identifican con una determinada morfología, estableciendo un sector dentro de la red sin segmentar (RSS). En total, se pueden diferenciar 4 sectores, cada uno con la misma demanda total. La segunda red estudiada se muestra en la Figura 3, y corresponde al sistema de distribución de agua de la localidad de Huerta Grande, provincia de Córdoba, Argentina. Esta red está compuesta por 431 nodos, 399 tubos y 5 tanques. Presenta una diferencia de cota de 270 metros entre la ubicación de los nodos de mayor y menor altura.

\section{Resultados}

Para la red de 25 nodos se obtienen diferentes sectorizaciones según el valor $\lambda$ utilizado. En la Figura 1-centro se observa el resultado de la optimización para $\lambda=0.5 \mathrm{y}$, en la Figura 1-derecha, para $\lambda=1.0$. En ambos casos, la máxima modularidad se obtiene realizando 8 cortes que están representados en la Figura 1 con válvulas de aislación. Estos cortes delimitan tres comunidades autónomas (módulos). Sin embargo, la distribución de los mismos es diferente para cada valor de $\lambda$ adoptado.

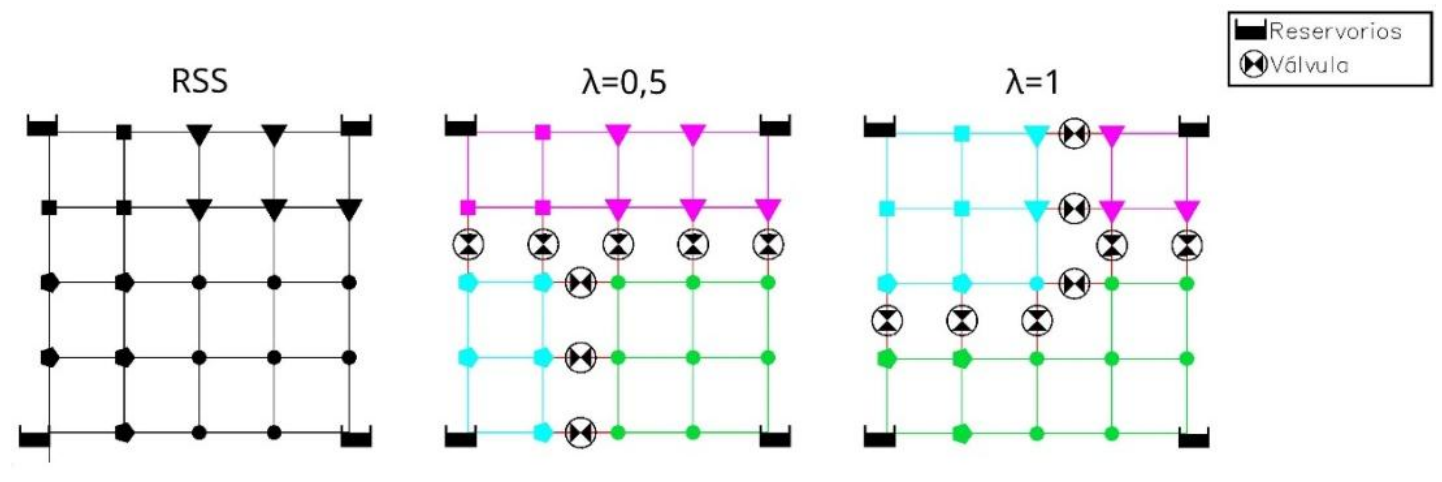

Figura 1: Red de 25 nodos. Izquierda, red sin segmentar (RSS). Centro y derecha, segmentación óptima para dos valores del parámetro de sintonía $\lambda$ de la función objetivo.

Cada una de las soluciones obtenidas tras la perturbación de las demandas nodales, en la red de 25 nodos, se muestra en la Figura 2. Se observa que la red es capaz de soportar grandes incrementos en las demandas cuando se afecta a un solo nodo $(\mathrm{N}=1)$ manteniendo aproximadamente constante su valor de resiliencia. Sin embargo, cuando se incrementa el número de nodos $(\mathrm{N}=10)$, la resiliencia muestra un decrecimiento sostenido. Sorprendentemente, la RSS posee la menor resiliencia cuando se la compara con las redes sectorizadas. Nótese, además, que la red sectorizada con $\lambda=0.5$; es aquella que mejor soporta los incrementos en las demandas cuando se afecta tanto a uno $(\mathrm{N}=1)$ como a diez nodos $(\mathrm{N}=10)$. 

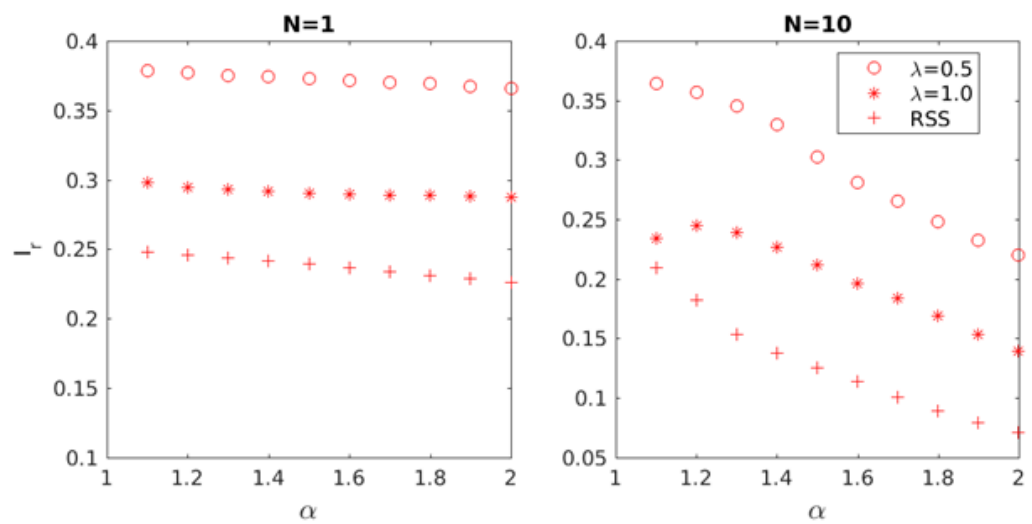

Figura 2: Resiliencia en función del parámetro de incremento en las demandas. Red de 25 nodos, resultados para un nodo (N=1) y diez nodos afectados $(\mathrm{N}=10)$.

El resultado de la sectorización para el segundo caso de estudio, la red de Huerta Grande, se muestra en la Figura 3. La igual ponderación de resiliencia e índice de modularidad $(\lambda=0.5)$ provee 4 comunidades, mientras que la sectorización con $\lambda=1.0$ provee solo 3 comunidades. En la Figura 4, se muestran el comportamiento de la red sectorizada para incrementos en las demandas. Al igual que en la red de 25 nodos, la peor confiabilidad se presenta en la RSS. Por otro lado, se observa un aumento en la resiliencia bajo los dos esquemas de segmentación, siendo la mejor respuesta aquella que se obtiene al considerar modularidad y resiliencia en la función objetivo.

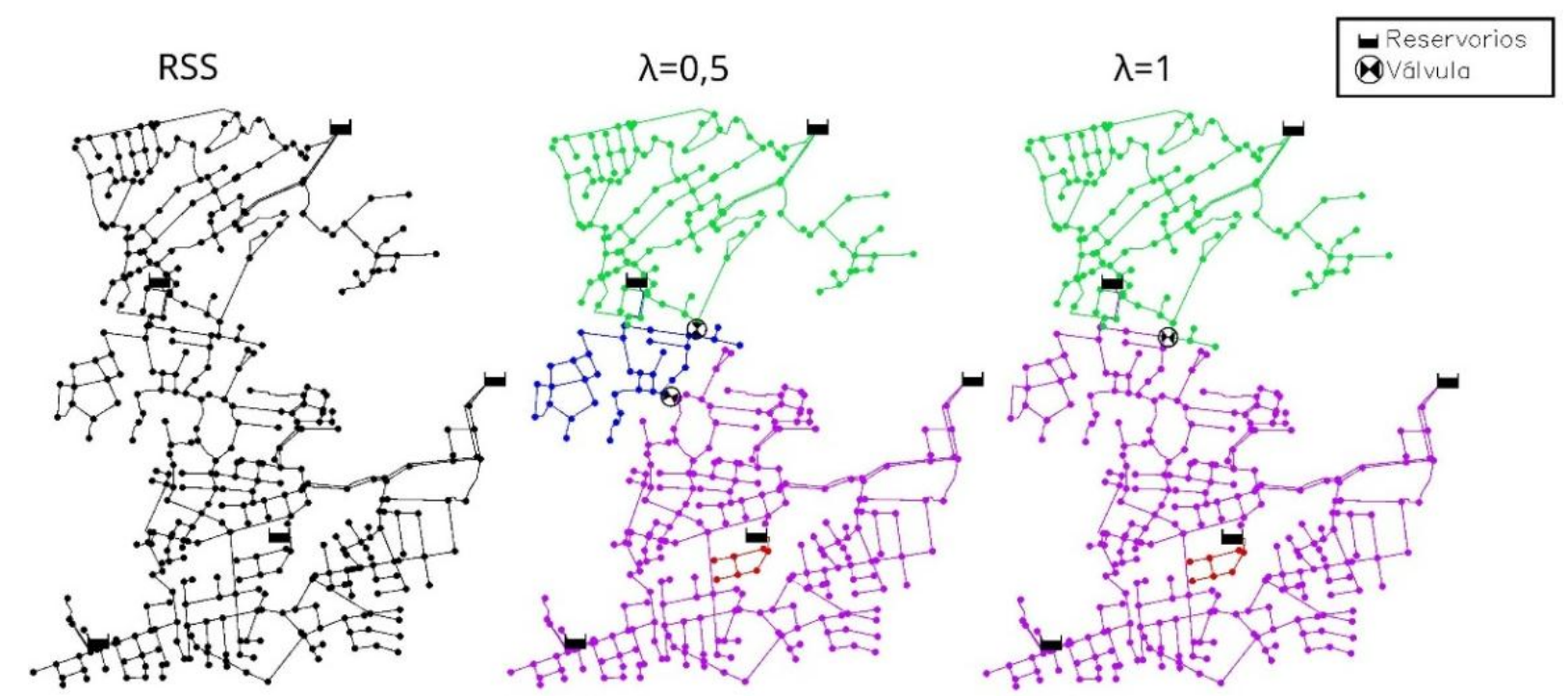

Figura 3: Red de Huerta Grande. Izquierda, red sin segmentar (RSS). Centro y derecha, segmentación óptima obtenida para dos valores del parámetro de ajuste $\lambda$ de la función objetivo. 

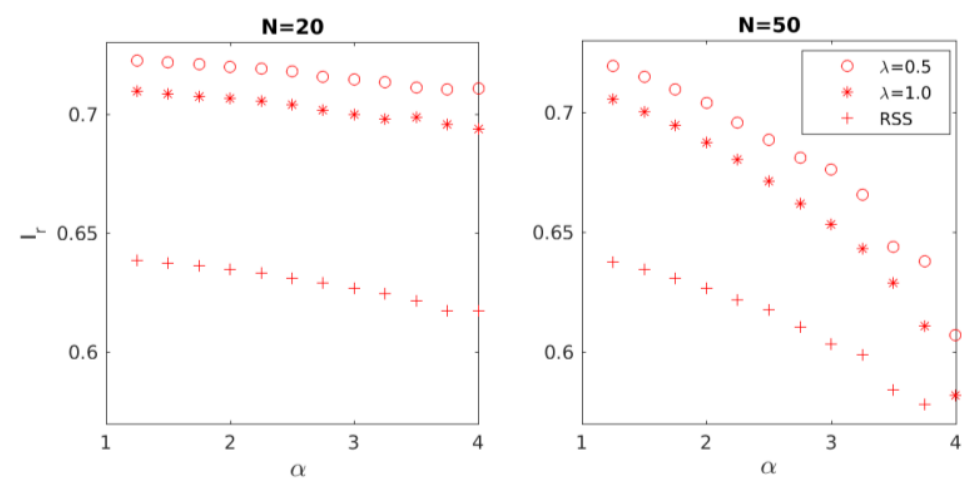

Figura 4: Red de Huerta Grande, resultados para 20 y 50 nodos afectados.

\section{Conclusiones}

En este trabajo se analizó la confiabilidad de la sectorización bajo incrementos de las demandas para dos casos de estudio: una red académica simple de 25 nodos, y una red real de la localidad de Huerta Grande, provincia de Córdoba. Desde un punto de vista operacional, los resultados muestran que la sectorización puede aumentar el rendimiento de la red si es realizada de manera óptima. Además, la sectorización basada en la modularidad topológica y el índice de resiliencia resultó ser la más eficiente para el sistema. Desde esta perspectiva, el diseño de la sectorización puede ser considerado como una herramienta práctica que ayuda a la toma de decisiones en el proceso de instalación de los dispositivos de aislación. Sin embargo, aunque el índice de resiliencia suele utilizarse como una medida de la confiabilidad del sistema hidráulico, no está claro que este índice pueda capturar otros aspectos de la confiabilidad que pueden afectar a los consumidores como la robustez frente a fallas mecánicas de la red.

\section{Referencias}

Farley, M. (2001). World Health Organization. Leakeage Management and Control: A Best Practice Training Manual. World Health Organization: Genova, Switzerland.

Castro-Gama M., Pan Q., Jonoski A., y Solomatine D. (2016). A graph theorical sectorization approach for energy reduction in water distribution networks. Proc. Engineering, 154:19-26.

Liu H., Zhao M., Zhang C. y Fu G. (2018). Comparing Topological Partitioning Methods for District Metered Areas in the Water Distribution Network. Water, 10:368.

Laucelli D., Simone, A., Berardi L. y Giustolisi O. (2017). Design of District Metering Areas for the Reduction of Leakages. J. Water Resour. Plann. Manage, 143:6.

Herrera M., Abraham E. y Stoianov I. (2015). Procedia Engineering, 119:1241-1248.

Kirkpatrick S., Gelatt J., y Vecchi M. (1983). Optimization by simulated annealing. Science, 220:671-680.

Newman M. y Girvan M. (2004). Finding and evaluating community structure in networks. Phys. Rev. E., 69(2).

Todini E. (2000). Looped water distribution networks design using a resilience index based heuristic approach. Urban Water, $2: 115-122$ 